\title{
Subject Index, Vol. 19
}

Angioma 196 Apert syndrome 151 Arachnoiditis 250 Aseptic meningitis 276 Astrocytoma 57 -, low grade 113 Axis fractures 225

Bacterial meningitis 276

Basal ganglia, germ cell tumors 121

Benign intracranial hypertension 206

Blindness 260

Brain anatomy 14

neoplasms 6

tumor(s) 57, 127, 171

- -, chemotherapy 171

-, infancy 180

-, irradiation 171

Cavernous angioma 196

Cerebrospinal fluid shunting 151,233

Cervicomedullary compression, separate atlantal lateral mass 165

Chiari malformation 143

Cloacal exstrophy 25

Communication problems, physician-patient-parent 104

Congenital craniovertebral anomalies 165

Cranial base 73

fossa, posterior 31 Craniofacial hydrocephalus 63

surgery 73

--, orbital reconstruction 73 Craniopharyngioma, radical resection 6 Craniosynostosis 63, 73 Craniovertebral junction 165 Cushing’s disease 6

Dermoid, filum terminale 81 Desmoplasia 57

Desmoplastic tumors, immunocytochem-istry 57

-, ultrastructure 57 Detrusor hyperreflexia 256

- sphincter dyssynergia 256

Endoscopic ventricular surgery 127

- - -, laser 127 Epidural hematoma 78 Epilepsy surgery, adolescents 40

- - children 40

- -, complications 40

Extra-axial brain fluid collections 202 Extracorporeal membrane oxygenation 202 Extradural hematoma 31

Fibrous dysplasia, childhood 260 Flow-regulating shunt system 156

Ganglioglioma 57

Germ cell tumor 121

Glial fibrillary acidic protein 21

Gliofibroma 57

Glioma 196

Halo immobilization, pediatric axis fractures 225 Headaches, slit-ventricle syndromes 15 Hydrocephalus 15,151,156,209,233 -, arrested 101 -, compensated 101 -, neuroendoscopy 127 Hydromyelia 243

Imperforate anus 25 Infratentorial tumor 180 Injury variables 135 Intracranial teratomas 84

-, prenatal diagnosis 84

-, resection 84

-, ultrasound 84 Isolated fourth ventricle 209

Lateral canthus 73

Lipomeningocele 250

-, early surgery 256

-, urodynamic studies 256

Lipomyelomeningocele 25, 243

Low-grade astrocytoma 186

- gliomas, chemotherapy 113

Lumbar disc 250

Mechanical shunt complications 156 Minor head injury, risk factors 135 Myelocystocele 25 Myelomeningocele 81, 243 Myeloschisis, immunohistochemistry 21

Neoplasms, children 196 Neurocysticercosis, magnetic resonance

imaging 206 Neurofibromatosis 186 Neuron-specific enolase 21 Nucleus gracilis 143 
Obex 143

Optic chiasm 6, 186

nerve 186

- - decompression 34

- $\quad$ - injury, conservative management 34

- pathway/hypothalamic gliomas, radiotherapy 186

Pediatric C-2 fractures 225

Peritoneal catheter insertion, laparoscopic

guidance 109 Poloxamer 407, leptomeningeal adhesion

prevention 250 Posterior fossa surgery, aseptic meningitis

syndrome 276

Rostrocaudal herniation 209

Scalp hamartoma, extracranial pathology 89

-, identical twins 89 Scaphocephaly 63 Shunt infection 233

- obstruction 156,233 Skull base surgery 260 Slit-ventricle syndrome, shunts 15 Spinal cord injury 225

- lipoma 243

stabilization 225 Steroids 276 Supratentorial tumor 180

'Talk and die' event 135 Tethered cord 25,243,250

- -, CO2 laser treatment 243

- - - dermoid tumors 243 Thalamus, germ cell tumors 121 Trapped fourth ventricle 209

Ultrasound, head 202

Vascular malformations, children 196 Vater association, tethered spinal cord 25 Ventricular tumors 127 Ventriculomegaly 151 Ventriculoperitoneal shunt 78, 109 Visual evoked potential 34 